\section{Contrast-enhanced fat-suppressed FLAIR for the characterization of leptomeningeal inflammation in optic neuritis}

\author{
Luis Pino-Lopez, Holger Wenz, Johannes Böhme, Máté Maros, Frank Schlichtenbrede, \\ Christoph Groden and Alex Förster
}

\section{Abstract}

Background: Leptomeningeal contrast enhancement on fluid-attenuated inversion recovery (FLAIR) images has been reported in patients with multiple sclerosis and interpreted as a biomarker of inflammation. In this study, we sought to evaluate this phenomenon in patients with optic neuritis (ON).

Methods: A total of 42 patients with suspected $O N$ were included in this prospective study and underwent a dedicated study magnetic resonance imaging (MRI) protocol including native and contrast-enhanced fat-suppressed thin-section axial and coronal FLAIR images on an $1.5 \mathrm{~T}$ magnetic resonance (MR) system.

Results: After diagnostic workup, 34 patients with final diagnosis of ON were analyzed in detail. On contrast-enhanced fat-suppressed FLAIR images, 25 (73.5\%) patients with ON demonstrated perioptic leptomeningeal enhancement, and in $3(8.8 \%)$ patients, this was even the only pathological MRI finding. In comparison, patients with perioptic leptomeningeal enhancement on contrast-enhanced fat-suppressed FLAIR images had a higher prevalence of additional hyperintense brain lesions $(p=0.022)$ as well as cerebrospinal fluid (CSF)-specific oligoclonal bands $(p=0.013)$ than patients without.

Conclusion: Perioptic leptomeningeal contrast enhancement on fat-suppressed FLAIR images is a novel marker in $\mathrm{ON}$ and possibly reflects a leptomeningeal inflammatory process preceding or accompanying ON. Thin-section contrast-enhanced fat-suppressed FLAIR images might be a useful addition in MRI protocols for patients with suspected ON.

Keywords: Contrast-enhanced, fat-suppressed, FLAIR, leptomeningeal inflammation, optic neuritis

Date received: 4 October 2017; revised: 1 March 2018; accepted: 14 March 2018

\section{Introduction}

Optic neuritis (ON) is related to an inflammatory demyelination of the optic nerve that predominantly affects young adults and usually presents as a painful unilateral impairment of vision. ${ }^{1} \mathrm{ON}$ may occur in isolation or be the first clinical sign of multiple sclerosis (MS). Furthermore, ON frequently occurs in MS patients in the clinical course. ${ }^{1}$ Usually, magnetic resonance imaging (MRI) is used in the evaluation of patients with suspected $\mathrm{ON}$ as it very sensitive in detecting the symptomatic lesion ${ }^{2}$ and useful to check for associated asymptomatic white matter lesions. ${ }^{3}$ Besides contrastenhanced fat-suppressed T1-weighted images, fluidattenuated inversion recovery (FLAIR) images have additional value in evaluating the optic nerve., ${ }^{4,5}$
Moreover, contrast-enhanced FLAIR images are much more sensitive than T1-weighted images for detecting low concentrations of gadolinium in the cerebrospinal fluid (CSF). ${ }^{6}$ In particular, in diseases affecting the leptomeningeal compartment, contrast-enhanced FLAIR images have been shown to be useful to demonstrate the leptomeningeal pathology such as bacterial meningitis, meningeal carcinomatosis, and meningeal angiomatosis. $^{7-11}$ In 2004, the hyperintense acute reperfusion marker was described in acute ischemic stroke for the first time. ${ }^{12}$ The phenomenon is caused by blood-brain barrier disruption following acute recanalization, reperfusion, and consecutive delayed gadolinium contrast enhancement in the subarachnoid space on FLAIR
Multiple Sclerosis Journal 2019, Vol. 25(6) 792-800 DOI: $10.1177 /$ 1352458518770268

(C) The Author(s), 2018. Article reuse guidelines: sagepub.com/journalspermissions
Correspondence to: A Förster

Department of

Neuroradiology,

Universitätsmedizin Mannheim, Heidelberg University, Theodor-KutzerUfer, Mannheim 68167 , Germany. alex.foerster@umm.de Luis Pino-Lopez Frank Schlichtenbrede Department of Ophthalmology, Universitätsmedizin Mannheim, Heidelberg University, Mannheim, Germany

Holger Wenz Johannes Böhme Máté Maros Christoph Groden Alex Förster Department of Neuroradiology, Universitätsmedizin Mannheim, Heidelberg University, Mannheim, Germany 
images. Meanwhile, leptomeningeal contrast enhancement on FLAIR images has also been reported in patients with MS and interpreted as a biomarker of inflammation, ${ }^{13,14}$ and there is increasing evidence that leptomeningeal inflammation might play an important role in the development of cerebral cortical gray matter pathology in MS. ${ }^{15}$ Protein accumulation, antigen presentation, and stimulation of reactive cells may promote a sustained leukocyte presence in the brain resulting in the formation of ectopic lymphoid-like tissue. ${ }^{16}$ While this has been primarily described in patients with primary- or secondary-progressive MS, ${ }^{15,16}$ signs of leptomeningeal inflammation on contrast-enhanced FLAIR images have been reported in approximately $20 \%$ of patients with relapsing-remitting MS. ${ }^{13,14}$ As Absinta et al. demonstrated, the histopathological basis of these MRI findings is leptomeningeal perivascular inflammation including T cells, B cells, and macrophages. ${ }^{13}$

However, so far no reports have been published on contrast enhancement in the perioptic subarachnoid space on FLAIR images in patients with ON. We therefore conducted a prospective study with special emphasis on this finding and here report the prevalence and distribution of perioptic leptomeningeal enhancement on contrast-enhanced fat-suppressed FLAIR images in patients with $\mathrm{ON}$ as well as clinical and laboratory associations.

\section{Methods}

\section{Patients and controls}

The study received approval (2015-552N-MA) from the institutional review board (IRB), and all participants provided written informed consent. After completing enrollment of patients with $\mathrm{ON}$, the protocol was amended and again approved by the IRB for enrollment of additional control subjects without ON.

In the study period, 42 patients with clinically suspected $\mathrm{ON}$ were included in the study. The diagnosis was based on clinical symptoms at presentation and during hospitalization corroborated by imaging and laboratory findings. Eight patients were excluded from the analysis due to a final diagnosis other than ON. In addition, the patients' brain MRI and laboratory test results were reviewed with special focus on acute and chronic white matter lesions and CSF findings, respectively (CSF-specific oligoclonal bands, CSF IgG index, CSF pleocytosis (white blood count (WBC) $>5 / \mathrm{mm}^{3}$ ), and CSF protein elevation (protein $>45 \mathrm{mg} / \mathrm{dL})$ ). After diagnostic workup, patients were diagnosed either with MS according to the 2010 revision of the McDonald criteria, with clinically isolated syndrome, or, if no additional white matter lesions were present, with isolated $\mathrm{ON}$.

The control group consisted of patients who underwent MRI for different reasons such as suspected ischemic stroke, follow-up of untreated or treated intracranial aneurysms, or follow-up after surgery of acoustic neurinoma, glioma, meningioma, or arteriovenous malformations. None of these patients had a history of $\mathrm{ON}$ or demyelinating disease.

\section{MRI}

All patients underwent MRI performed on a $1.5 \mathrm{~T}$ scanner (Magnetom Avanto; Siemens Medical Systems, Erlangen, Germany) using a standardized MRI protocol including (1) transverse, coronal, and sagittal localizing sequences; axial (2) T1-weighted images; (3) FLAIR images; followed by thin-section ( $3 \mathrm{~mm}$ ) axial (4) fatsuppressed T1-weighted images; (5) fat-suppressed FLAIR images (field of view $230 \mathrm{~mm} \times 169 \mathrm{~mm}$, matrix $256 \mathrm{~mm} \times 164 \mathrm{~mm}$, number of slices 24 , TR $9000 \mathrm{~ms}$, TE $89 \mathrm{~ms}$, TI $2500 \mathrm{~ms}$, spectral fat saturation, acquisition time: 2:26minutes:seconds); as well as thin-section (3 mm) coronal (6) fat-suppressed T1-weighted images; (7) T2-weighted images; and (8) fat-suppressed FLAIR images (field of view $230 \mathrm{~mm} \times 169 \mathrm{~mm}$, matrix $256 \mathrm{~mm} \times 168 \mathrm{~mm}$, number of slices 24 , TR $9000 \mathrm{~ms}$, TE $89 \mathrm{~ms}$, TI $2500 \mathrm{~ms}$, spectral fat saturation, acquisition time: 2:26minutes:seconds). After manual injection of single-dose contrast agent (gadoteric acid (Dotarem; Guerbet, Aulnay-sous-Bois, France), (9) T1-weighted images identical to (2); (10) and (11) T1-weighted images identical to (4) and (6); as well as (12) and (13) fat-suppressed FLAIR images identical to (5) and (8) were completed. Correspondingly, thin-section axial and coronal fat-suppressed FLAIR images before and after intravenous contrast agent application were added to the routinely performed MRI protocol in the control group. The axial and coronal FLAIR sequences were acquired approximately 10 and 12 minutes after intravenous contrast agent application.

\section{MRI analysis}

All MRI scans were reviewed in consensus by two readers (L.P.-L. and A.F. with 1 and 11 years' experience in neuroimaging) with regard to morphology and signal characteristics in the optic nerve (swelling or increased signal intensity), lesion location (intraorbital, canalicular, or cranial part), enhancement in the optic nerve on contrast-enhanced T1-weighted images, perioptic leptomeningeal enhancement on contrastenhanced fat-suppressed FLAIR images, as well as acute white matter lesions on contrast-enhanced 
T1-weighted images and chronic white matter lesions on FLAIR images.

\section{Statistical analysis}

All statistical analyses were performed using Statistical Product and Service Solutions (SPSS) statistics for Windows (Release 17.0; SPSS, Chicago, IL, USA). Descriptive and clinical data was analyzed using $\chi^{2}$-based tests (sex, symptoms, diagnosis) or the Mann-Whitney $U$ test (age, duration of symptoms) as appropriate. MRI findings were analyzed using $\chi^{2}$ based tests (presence of T2 lesion, contrast enhancement, perioptic leptomeningeal enhancement, additional hyperintense brain lesions). CSF analysis results were analyzed using $\chi^{2}$-based tests (CSFspecific oligoclonal bands, CSF pleocytosis, CSF protein elevation) or the Mann-Whitney $U$ test (CSF IgG index) as appropriate. All statistics was performed with a 0.05 level of significance.

\section{Results}

\section{Patient demographics}

A total of 34 patients with final diagnosis of unilateral ON were analyzed in detail. The median age was 30 (IQR: 25-41.5) years; 7 (20.6\%) patients were male and $27(79.4 \%)$ patients were female. Among the ON patients, there were four $(11.8 \%)$ patients with a history of MS. Of the remaining 30 patients, further 6 (20\%) were diagnosed with MS according to the 2010 revision of the McDonald criteria, 14 (46.7\%) were diagnosed with clinically isolated syndrome, and 10 $(33.3 \%)$ were diagnosed with isolated ON. In none of the patients, diagnostic workup indicated other causes of $\mathrm{ON}$ such as autoimmune disorders or infectious diseases. Clinical symptoms included sudden loss of vision $(100 \%)$, pain with eye movement $(50 \%)$, and loss of color vision $(61.8 \%)$.

In the control group, 20 patients were included. The median age was 43.5 (IQR: 29-49.5) years; 6 (30\%) patients were male and $14(70 \%)$ patients were female.

\section{MRI findings}

MRI was performed within a median time of 3 (IQR: 1-7) days after onset of symptoms. Overall, a lesion in the optic nerve consistent with unilateral ON could be identified in $29(85.3 \%)$ patients on MRI. In 13 (38.2\%) patients, the lesion was located in the intraorbital part of the optic nerve; in $5(14.7 \%)$ patients in the intraorbital and canalicular part; in $2(5.9 \%)$ patients in the canalicular part; in $5(14.7 \%)$ patients in the canalicular and cranial part; and in $4(11.8 \%)$ patients in the cranial part of the optic nerve. On contrast-enhanced fat-suppressed FLAIR images, 25 (73.5\%) patients with ON demonstrated perioptic leptomeningeal enhancement (see Figures 1 and 2), and in $3(8.8 \%)$ patients this was even the only pathological finding (see Figure 3). A perioptic leptomeningeal enhancement was more frequently found on coronal FLAIR images $(100 \%)$ compared to axial FLAIR images (13/25 (52\%)). In the contralateral optic nerve, none of the ON patients demonstrated perioptic leptomeningeal enhancement on contrast-enhanced fatsuppressed FLAIR images (see Figures 1 and 2). In 19 (55.9\%) patients, a pathological hyperintense signal or swelling of the optic nerve could be demonstrated on T2-weighted images. A pathological enhancement in the optic nerve on contrast-enhanced T1-weighted images was found in $23(67.6 \%)$ patients. Of the 25 patients with perioptic leptomeningeal enhancement, $16(64 \%)$ patients demonstrated signal alterations in the optic nerve on T2-weighted images as well as contrast enhancement on T1-weighted images, $6(24 \%)$ patients demonstrated only contrast enhancement in the optic nerve on T1-weighted images, while the remaining $3(12 \%)$ patients demonstrated neither signal alterations on T2-weighted images nor contrast enhancement on T1-weighted images. Of the nine patients without perioptic leptomeningeal enhancement, three (33.3\%) patients demonstrated only signal alterations in the optic nerve on T2-weighted images, one $(11.1 \%)$ patient demonstrated only contrast enhancement in the optic nerve on T1-weighted images, while the remaining five $(55.6 \%)$ patients demonstrated neither signal alterations on $\mathrm{T} 2$-weighted images nor contrast enhancement on $\mathrm{T} 1$-weighted images. In ON patients with signal alterations or swelling in the optic nerve, symptom duration was significantly longer than in patients without $(3(2-7)$ vs 2 $(0-5) ; p=0.036)$. Regarding the patients with and without perioptic leptomeningeal contrast enhancement on fat-suppressed FLAIR images $(p=0.23)$ or contrast enhancement in the optic nerve on contrastenhanced T1-weighted images $(p=0.44)$, no differences in symptom durations could be found. Additional chronic white matter lesions on FLAIR images were observed in $22(64.7 \%)$ patients, whereas acute white matter lesions with a pathological enhancement on contrast-enhanced T1-weighted images were found in only $1(2.9 \%)$ patient.

In the control group, none of the patients demonstrated perioptic leptomeningeal enhancement on contrast-enhanced fat-suppressed FLAIR images (see Figure 4.) 


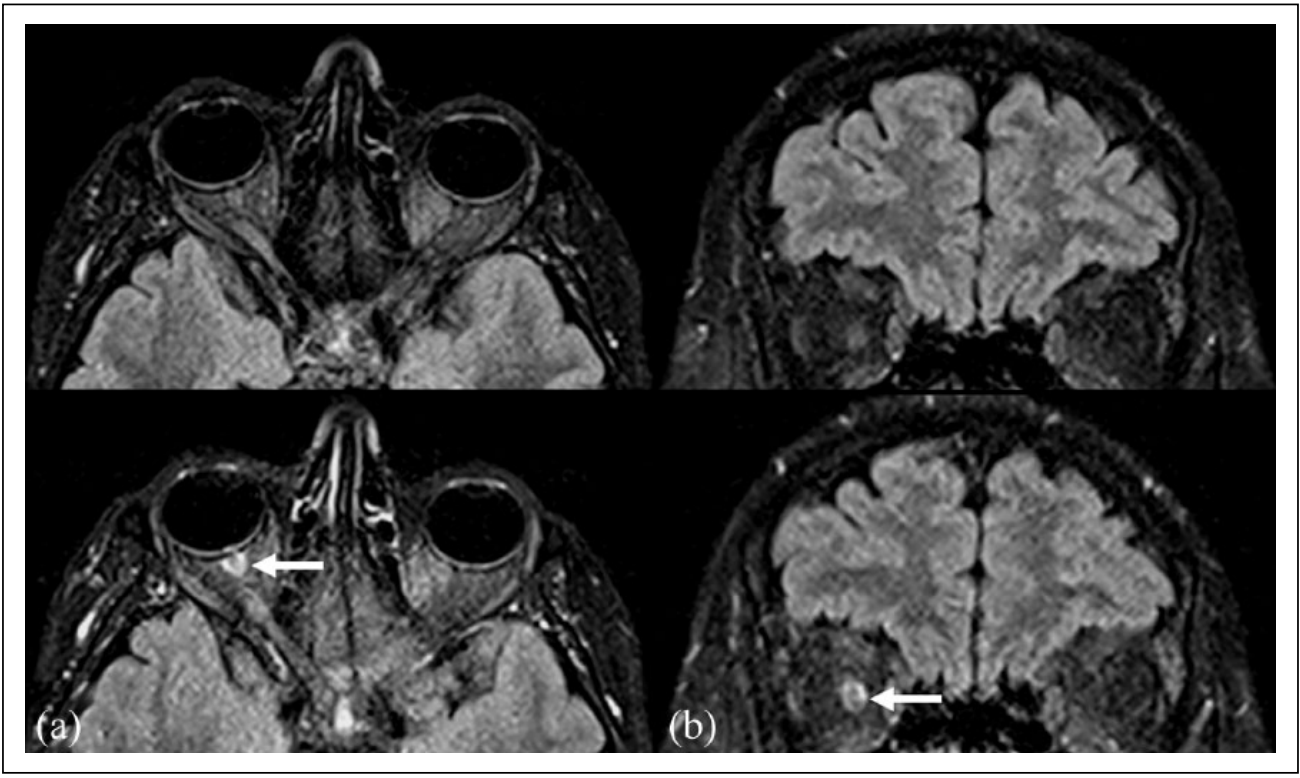

Figure 1. Perioptic leptomeningeal enhancement in a 38-year-old patient with optic neuritis. In comparison, (a) native (upper row) and contrast-enhanced fat-suppressed thin-section axial FLAIR images (lower row) as well as (b) native (upper row) and contrast-enhanced fat-suppressed thin-section coronal FLAIR images (lower row) show perioptic leptomeningeal enhancement (arrows). The contralateral optic nerve is unremarkable.

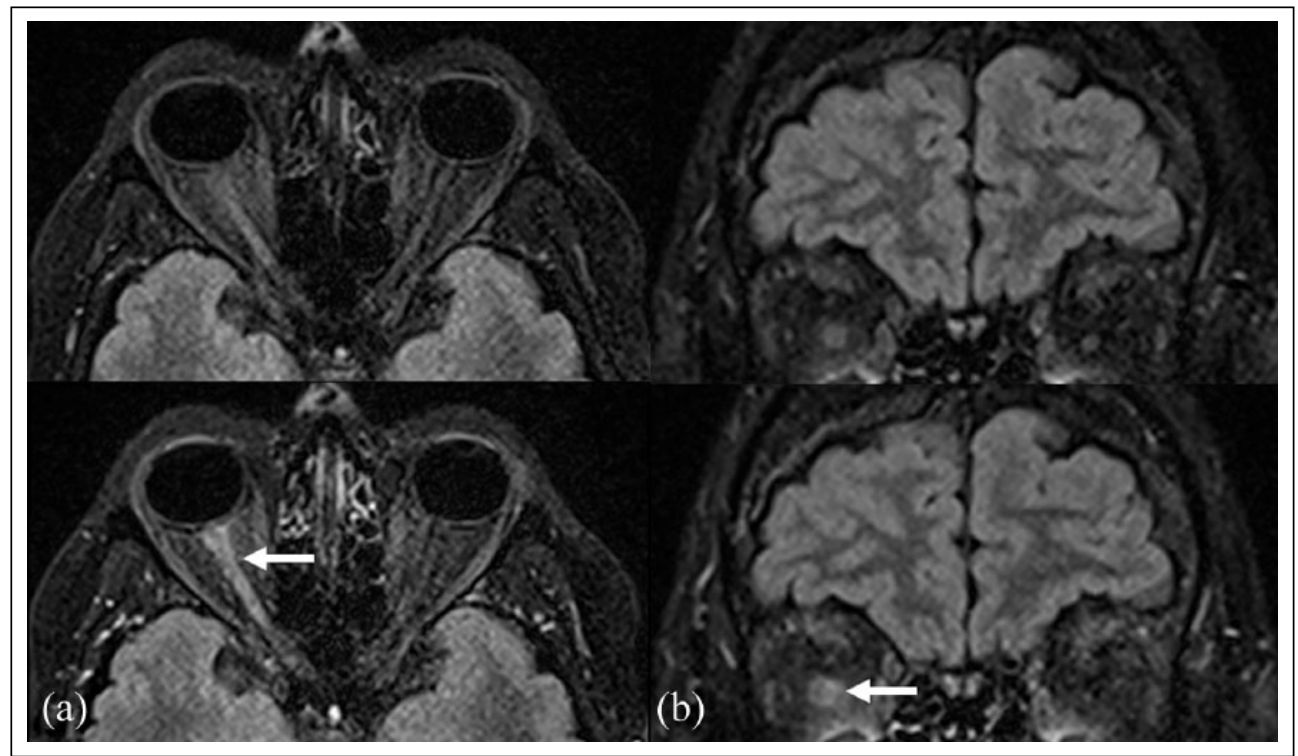

Figure 2. Extensive perioptic leptomeningeal enhancement in a 37-year-old patient with optic neuritis. In comparison, (a) native (upper row) and contrast-enhanced fat-suppressed thin-section axial FLAIR images (lower row) as well as (b) native (upper row) and contrast-enhanced fat-suppressed thin-section coronal FLAIR images (lower row) show perioptic leptomeningeal enhancement (arrows). The contralateral optic nerve is unremarkable.

In comparison, patients with perioptic leptomeningeal enhancement on contrast-enhanced fat-suppressed FLAIR images had a higher prevalence of additional hyperintense brain lesions $(p=0.022)$ as well as CSFspecific oligoclonal bands $(p=0.013)$ than patients without. With regard to clinical presentation, duration of symptoms, as well as CSF IgG index, pleocytosis, and protein, no significant differences were observed between both groups (see Tables 1 and 2 for details). Regarding the patients who demonstrated perioptic leptomeningeal enhancement on contrast-enhanced fat-suppressed FLAIR images as the only pathological 


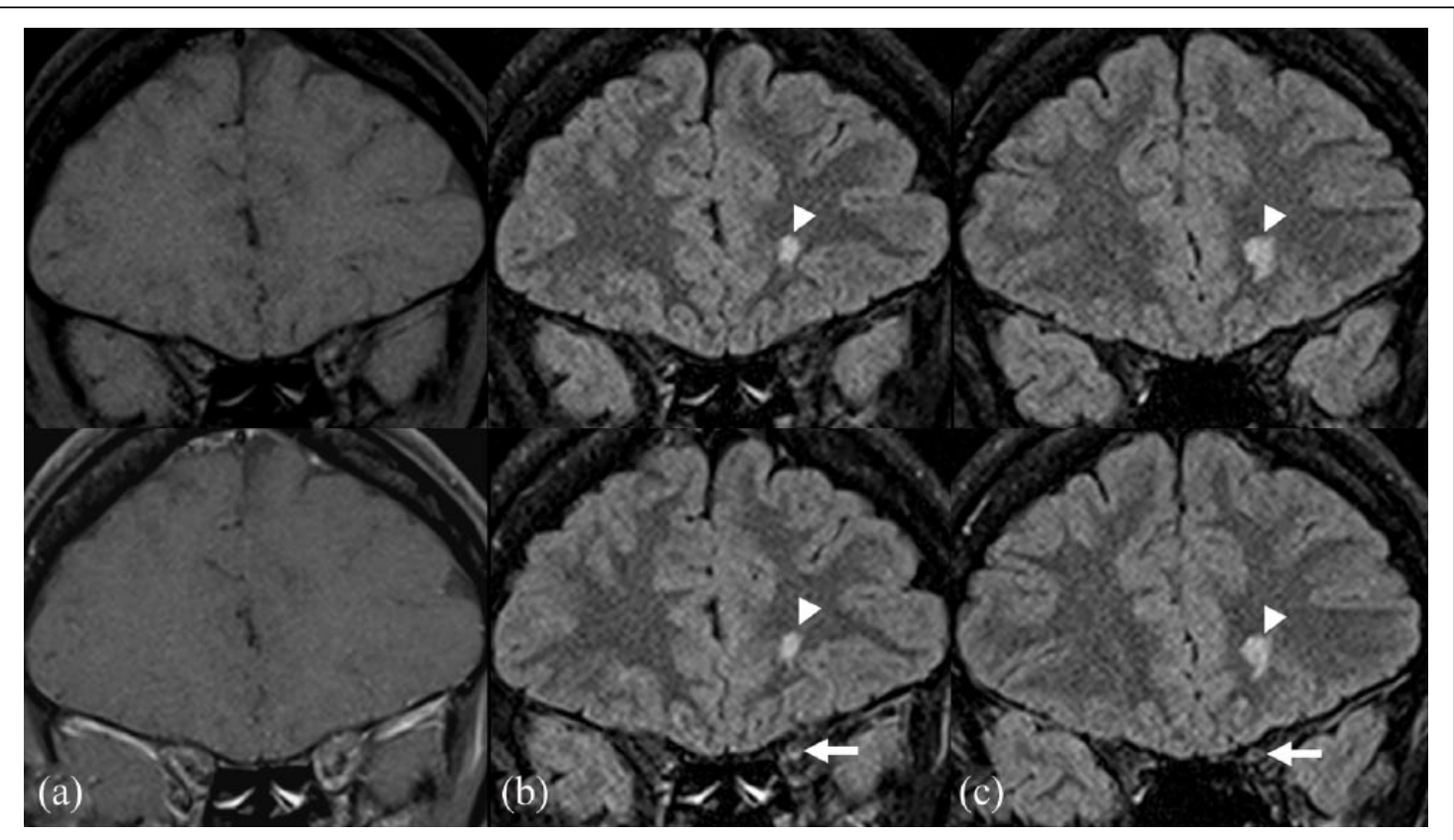

Figure 3. Perioptic leptomeningeal enhancement as the only pathological finding in a 30-year-old woman with optic neuritis. In comparison, (a) native (upper row) and contrast-enhanced (lower row) coronal T1-weighted images reveal no contrast enhancement in the left optic nerve. In comparison, (b) and (c) native (upper row) and contrast-enhanced fat-suppressed thin-section coronal FLAIR images (lower row) in two consecutive planes demonstrate perioptic leptomeningeal enhancement as the only pathological finding (arrows). Note the chronic white matter lesion in the left hemisphere (arrowhead).

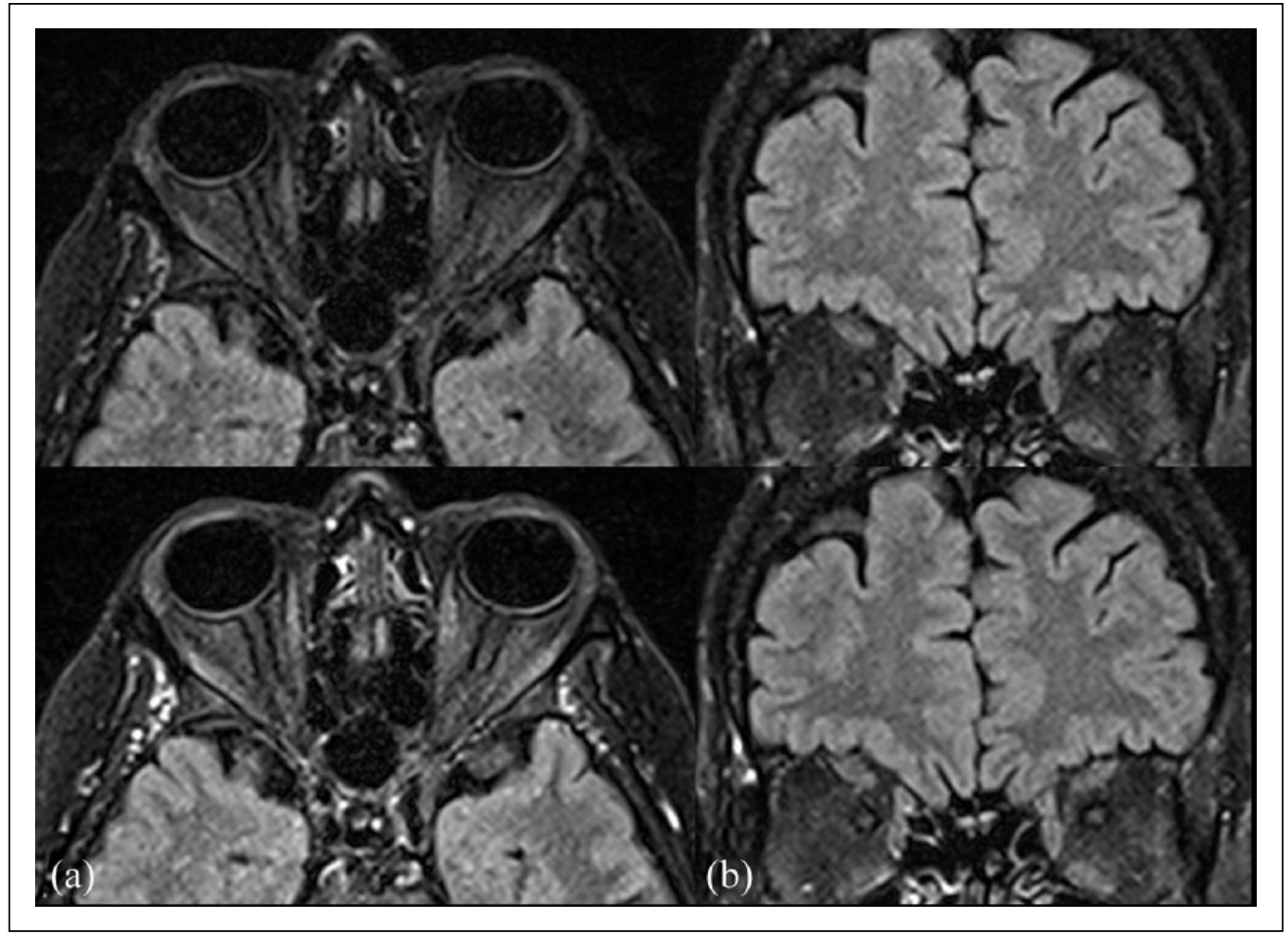

Figure 4. The optic nerve in a 56-year-old control subject. In comparison, (a) native (upper row) and contrast-enhanced fat-suppressed thin-section axial FLAIR images (lower row) as well as (b) native (upper row) and contrast-enhanced fatsuppressed thin-section coronal FLAIR images (lower row) show no perioptic leptomeningeal enhancement. 
Table 1. Characteristics of optic neuritis patients with perioptic leptomeningeal enhancement on contrast-enhanced fatsuppressed FLAIR images.

\begin{tabular}{|c|c|c|c|c|}
\hline & \multirow[t]{2}{*}{ All, $n=34$} & \multicolumn{2}{|l|}{ Contrast enhancement } & \multirow[t]{2}{*}{$p$ value } \\
\hline & & Yes, $n=25$ & $\mathrm{No}=9$ & \\
\hline Age (years), median (IQR) & $31(25-42.5)$ & $30(25-41)$ & $32(23-44)$ & 0.97 \\
\hline Male sex, $n(\%)$ & $7(20.6)$ & $4(16)$ & $3(33.3)$ & 0.27 \\
\hline $\begin{array}{l}\text { Duration of symptoms (days), } \\
\text { median (IQR) }\end{array}$ & $3(1-7)$ & $3(2-7)$ & $2(0.5-4)$ & 0.23 \\
\hline \multicolumn{5}{|l|}{ Clinical presentation } \\
\hline Sudden loss of vision, $n(\%)$ & $34(100)$ & $25(100)$ & $9(100)$ & NA \\
\hline Pain with eye movement, $n(\%)$ & $17(50)$ & $12(48)$ & $5(55.6)$ & 0.70 \\
\hline Loss of color vision, $n(\%)$ & $21(61.8)$ & $15(60)$ & $6(66.7)$ & 0.72 \\
\hline \multicolumn{5}{|l|}{ MRI findings } \\
\hline $\mathrm{T} 2$ lesion in the optic nerve, $n(\%)$ & $19(55.9)$ & $16(64)$ & $3(33.3)$ & 0.11 \\
\hline $\begin{array}{l}\text { Contrast enhancement in the } \\
\text { optic nerve, } n(\%)\end{array}$ & $23(67.6)$ & $22(88)$ & $1(11.1)$ & $<0.001$ \\
\hline $\begin{array}{l}\text { Additional hyperintense brain } \\
\text { lesions, } n(\%)\end{array}$ & $22(64.7)$ & $19(76)$ & $3(33.3)$ & 0.022 \\
\hline \multicolumn{5}{|l|}{ Diagnosis } \\
\hline Isolated optic neuritis, $n(\%)$ & $10(29.4)$ & $5(20)$ & $5(55.6)$ & 0.13 \\
\hline Clinically isolated syndrome, $n(\%)$ & $14(41.2)$ & $12(48)$ & $2(22.2)$ & \\
\hline Multiple sclerosis, $n(\%)$ & $10(29.4)$ & $8(32)$ & $2(22.2)$ & \\
\hline \multicolumn{5}{|l|}{ CSF analysis, available in 25} \\
\hline Oligoclonal bands, $n(\%)$ & $15(60)$ & $14(73.7)$ & $1(16.7)$ & 0.013 \\
\hline IgG index, median (IQR) & $0.62(0.56-0.9825)$ & $0.645(0.575-1.4675)$ & $0.575(0.45-0.7025)$ & 0.077 \\
\hline Pleocytosis, $n(\%)$ & $12(48)$ & $11(57.9)$ & $1(16.7)$ & 0.078 \\
\hline Protein elevation, $n(\%)$ & $6(24)$ & $3(15.8)$ & $3(50)$ & 0.087 \\
\hline
\end{tabular}

finding, two of these showed additional hyperintense brain lesions on FLAIR images and were diagnosed with clinically isolated syndrome. One of these patients also had CSF-specific oligoclonal bands.

\section{Discussion}

In this study, we describe two novel and essential findings in ON: (1) a perioptic contrast enhancement on contrast-enhanced fat-suppressed FLAIR images is present in more than $70 \%$ of patients with $\mathrm{ON}$ and (2) in some cases, this perioptic contrast enhancement may be the only pathological finding. Thus, the perioptic contrast enhancement on fat-suppressed FLAIR images might indicate that the inflammatory process in $\mathrm{ON}$ is not only restricted to the optic nerve but may also affect the leptomeningeal compartment. Furthermore, the observation of perioptic contrast enhancement as the only pathological finding in a subset of ON patients might imply that the inflammation possibly even started in the leptomeningeal compartment and spread to the optic nerve in the course of disease corresponding to the recently published observations in MS. Another explanation of perioptic leptomeningeal contrast enhancement might be the mere diffusion of contrast agent from a pronounced inflammation in the optic nerve to the adjacent perioptic leptomeningeal compartment. Nevertheless, this does not explain isolated perioptic leptomeningeal contrast enhancement in a subset of ON patients without contrast enhancement in the optic nerve in this study.

More than a decade ago, Hickman et al. ${ }^{17}$ already described optic nerve sheath enhancement in a subset of ON patients on triple-dose contrast-enhanced T1-weighted images. Enhancement of the optic nerve sheath was observed in approximately $75 \%$ of patients. Since then, this specific finding has not been in the focus of clinical studies but has been reported rather as a secondary finding in a few smaller studies on MRI in ON. ${ }^{18,19}$ However, the frequency of a perioptic enhancement is lower in the more recent studies than in the initial study. ${ }^{18}$ An explanation might be the 
Table 2. Characteristics of optic neuritis patients who underwent a lumbar puncture.

\begin{tabular}{|c|c|c|c|c|}
\hline & \multirow[t]{2}{*}{ All, $n=34$} & \multicolumn{2}{|c|}{ Lumbar puncture } & \multirow[t]{2}{*}{$p$ value } \\
\hline & & Yes, $n=25$ & $\mathrm{No}=9$ & \\
\hline Age (years), median (IQR) & $31(25-42.5)$ & $30(25-41.5)$ & $33(19.5-56)$ & 0.97 \\
\hline Male sex, $n(\%)$ & $7(20.6)$ & $6(24)$ & $1(11.1)$ & 0.41 \\
\hline Duration of symptoms (days), median (IQR) & $3(1-7)$ & $3(2-7)$ & $2(0.5-3)$ & 0.09 \\
\hline \multicolumn{5}{|l|}{ MRI findings } \\
\hline $\mathrm{T} 2$ lesion in the optic nerve, $n(\%)$ & $19(55.9)$ & $16(64)$ & $3(33.3)$ & 0.11 \\
\hline Contrast enhancement in the optic nerve, $n(\%)$ & $23(67.6)$ & $17(68)$ & $6(66.7)$ & 0.94 \\
\hline Perioptic leptomeningeal enhancement, $n(\%)$ & $25(73.5)$ & $19(76)$ & $6(66.7)$ & 0.59 \\
\hline Additional hyperintense brain lesions, $n(\%)$ & $22(64.7)$ & $18(72)$ & $4(44.4)$ & 0.14 \\
\hline \multicolumn{5}{|l|}{ Diagnosis } \\
\hline Isolated optic neuritis, $n(\%)$ & $10(29.4)$ & $5(20)$ & $5(55.6)$ & 0.06 \\
\hline Clinically isolated syndrome, $n(\%)$ & $14(41.2)$ & $13(52)$ & $1(11.1)$ & \\
\hline Multiple sclerosis, $n(\%)$ & $10(29.4)$ & $7(28)$ & $3(33.3)$ & \\
\hline
\end{tabular}

fact that triple-dose contrast-enhanced MRI is nowadays not generally recommended in patients with ON or $\mathrm{MS}^{20}$ resulting in a lower detection rate. Contrastenhanced FLAIR images in turn have the advantage of a considerably higher sensitivity for minute concentrations of gadolinium-based contrast agents in the subarachnoid space ${ }^{6}$ and therefore might complement MRI protocols when a special focus shall be laid upon the perioptic enhancement in ON. Interestingly, other studies described a more extensive inflammation of the perioptic soft tissue on contrast-enhanced T1-weighted images in up to one third of $\mathrm{ON}$ patients with antibodies against myelin oligodendrocyte glycoprotein. ${ }^{21,22}$ These patients differed significantly from the patients in this study with regard to clinical (most cases with recurrent or bilateral $\mathrm{ON}$ ) as well as radiological aspects (extensive involvement of the perioptic soft tissue, different white matter lesion pattern). Nevertheless, contrast-enhanced fat-suppressed FLAIR images could be useful for a more detailed characterization of the perineural inflammation in these ON patients as well.

Besides the above-mentioned pathophysiological considerations, and aspects concerning possible future studies, inclusion of contrast-enhanced fat-suppressed FLAIR images in routine MRI protocols could be a valuable addition in patients with suspected $O N$ in daily clinical practice since perioptic contrast enhancement on FLAIR images was a frequent and in some cases even the only pathological MRI finding in ON.

Contrast enhancement in the optic nerve has been observed in two thirds of ON patients in this study. It is thought to reflect the acute inflammation and blood-brain barrier impairment and has been demonstrated in up to $94.4 \%$ of ON patients in previous studies. ${ }^{2}$ However, in more recently published studies, the frequency of contrast enhancement ranged markedly lower between $66.7 \%$ and $85 \% .{ }^{17,18,23,24}$

Signal alterations or swelling in the optic nerve on T2-weighted images were observed in approximately half of the patients with ON in this study. This is also lower in comparison with previous studies reporting signal alterations or swelling in the optic nerve in $79.8 \%$ to $100 \%{ }^{17,18,24,25}$ Possibly, this observation can be attributed at least partly to the fact that in this study, ON patients underwent MRI at an early stage of disease within a median of 3 days after symptom onset which is earlier than in the other studies in which ON patients underwent MRI within a median of 5 days, ${ }^{24}$ 13 days, ${ }^{17,23}$ or 21 days. ${ }^{25}$ In another study, a range of 7-45 days from symptom onset to MRI has been reported. ${ }^{18}$ In MS lesions in the brain, Kermode et al..$^{26}$ showed that contrast enhancement may precede lesion appearance on T2-weighted images. Thus, the observed discrepancy between this study and previous studies may be explained by the different time points of MRI examination. This consideration might also be corroborated by the finding of this study that ON patients with signal alteration or swelling in the optic nerve had a significantly longer time from symptom onset to MRI compared to those ON patients without. However, to our best knowledge, there are no studies that investigated systematically the temporal evolution of optic nerve lesions in ON on MRI, and, regrettably, a follow-up MRI was not included in this study. 
This study has some limitations. First, this is a clinical study of moderate sample size. Nevertheless, to our knowledge, this is the first series investigating the additional value of leptomeningeal enhancement on contrast-enhanced fat-suppressed FLAIR images in ON. Second, we did not perform a follow-up MRI. Consequently, we cannot provide information on the course of perioptic leptomeningeal contrast enhancement in ON. Despite these limitations, the study has several strengths including the prospective study design with a standardized MRI protocol and MRI examinations on one scanner type.

Perioptic leptomeningeal contrast enhancement on FLAIR images is a novel marker in ON and possibly reflects a leptomeningeal inflammatory process preceding or accompanying ON. Consequently, inclusion of contrast-enhanced fat-suppressed FLAIR images in MRI protocols for patients with suspected $\mathrm{ON}$ might be a useful addition.

\section{Acknowledgements}

L.P.-L.: design of the study, analysis of the data, drafting, and revising the manuscript. H.W. and J.B.: acquisition of data and revising the manuscript. M.M.: acquisition of data, statistical analysis, and revising the manuscript. F.S. and C.G.: analysis of the data and revising the manuscript. A.F.: design of the study, acquisition of data, analysis of the data, drafting, and revising the manuscript.

\section{Declaration of Conflicting Interests}

The author(s) declared no potential conflicts of interest with respect to the research, authorship, and/or publication of this article.

\section{Funding}

The author(s) received no financial support for the research, authorship, and/or publication of this article.

\section{ORCID iD}

Alex Förster (iD https://orcid.org/0000-0002-86790060

\section{References}

1. Toosy AT, Mason DF and Miller DH. Optic neuritis. Lancet Neurol 2014; 13: 83-99.

2. Kupersmith MJ, Alban T, Zeiffer B, et al. Contrastenhanced MRI in acute optic neuritis: Relationship to visual performance. Brain 2002; 125: 812-822.

3. Beck RW, Arrington J, Murtagh FR, et al. Brain magnetic resonance imaging in acute optic neuritis:
Experience of the optic neuritis study group. Arch Neurol 1993; 50: 841-846.

4. McKinney AM, Lohman BD, Sarikaya B, et al. Accuracy of routine fat-suppressed FLAIR and diffusion-weighted images in detecting clinically evident acute optic neuritis. Acta Radiol 2013; 54: 455-461.

5. Boegel KH, Tyan AE, Iyer VR, et al. Utility of coronal contrast-enhanced fat-suppressed FLAIR in the evaluation of optic neuropathy and atrophy. Eur $J$ Radiol Open 2017; 4: 13-18.

6. Mamourian AC, Hoopes PJ and Lewis LD. Visualization of intravenously administered contrast material in the CSF on fluid-attenuated inversion-recovery MR images: An in vitro and animal-model investigation. AJNR 2000; 21: 105-111.

7. Mathews VP, Caldemeyer KS, Lowe MJ, et al. Brain: Gadolinium-enhanced fast fluid-attenuated inversion-recovery MR imaging. Radiology 1999; 211: 257-263.

8. Tsuchiya K, Katase S, Yoshino A, et al. FLAIR MR imaging for diagnosing intracranial meningeal carcinomatosis. AJR 2001; 176: 1585-1588.

9. Griffiths PD, Coley SC, Romanowski CA, et al. Contrast-enhanced fluid-attenuated inversion recovery imaging for leptomeningeal disease in children. AJNR 2003; 24: 719-723.

10. Galassi W, Phuttharak W, Hesselink JR, et al. Intracranial meningeal disease: Comparison of contrast-enhanced MR imaging with fluid-attenuated inversion recovery and fat-suppressed $\mathrm{T} 1$-weighted sequences. AJNR 2005; 26: 553-559.

11. McKinney A, Palmer C, Short J, et al. Utility of fat-suppressed FLAIR and subtraction imaging in detecting meningeal abnormalities. Neuroradiology 2006; 48: 881-885.

12. Latour LL, Kang DW, Ezzeddine MA, et al. Early blood-brain barrier disruption in human focal brain ischemia. Ann Neurol 2004; 56: 468-477.

13. Absinta M, Vuolo L, Rao A, et al. Gadoliniumbased MRI and characterization of leptomeningeal inflammation in multiple sclerosis. Neurology 2015; 85: 18-28.

14. Zivadinov R, Ramasamy DP, Vaneckova M, et al. Leptomeningeal contrast enhancement is associated with progression of cortical atrophy in MS: A retrospective, pilot, observational longitudinal study. Mult Scler 2016; 23: 1336-1345.

15. Howell OW, Reeves CA, Nicholas R, et al. Meningeal inflammation is widespread and linked to cortical pathology in multiple sclerosis. Brain 2011; 134: 2755-2771. 
Visit SAGE journals online journals.sagepub.com home/ms

( SAGE journals
16. Serafini B, Rosicarelli B, Magliozzi R, et al. Detection of ectopic B-cell follicles with germinal centers in the meninges of patients with secondary progressive multiple sclerosis. Brain Pathol 2004; 14: 164-174.

17. Hickman SJ, Miszkiel KA, Plant GT, et al. The optic nerve sheath on MRI in acute optic neuritis. Neuroradiology 2005; 47: 51-55.

18. Lim SA, Sitoh YY, Chng SM, et al. Magnetic resonance imaging in acute optic neuritis in Singapore. Ann Acad Med Singapore 2009; 38: 821-826.

19. Son DY, Park KA, Seok SS, et al. Initial pattern of optic nerve enhancement in Korean patients with unilateral optic neuritis. Korean J Ophthalmol 2017; 31: 71-79.

20. Traboulsee A, Simon JH, Stone L, et al. Revised recommendations of the consortium of MS centers task force for a standardized MRI protocol and clinical guidelines for the diagnosis and follow-up of multiple sclerosis. AJNR 2016; 37: 394-401.

21. Kim SM, Woodhall MR, Kim J-S, et al. Antibodies to MOG in adults with inflammatory demyelinating disease of the CNS. Neurol Neuroimmunol Neuroinflamm 2015; 2: e163.

22. Biotti D, Bonneville F, Tournaire E, et al. Optic neuritis in patients with anti-MOG antibodies spectrum disorder: MRI and clinical features from a large multicentric cohort in France. J Neurol 2017; 264: $2173-2175$.

23. Hickman SJ, Toosy AT, Miszkiel KA, et al. Visual recovery following acute optic neuritis: A clinical, electrophysiological and magnetic resonance imaging study. J Neurol 2004; 251: 996-1005.

24. Berg S, Kaschka I, Utz KS, et al. Baseline magnetic resonance imaging of the optic nerve provides limited predictive information on short-term recovery after acute optic neuritis. PLoS ONE 2015; 10: e0113961.

25. Soelberg K, Skejoe HPB, Grauslund J, et al. Magnetic resonance imaging findings at the first episode of acute optic neuritis. Mult Scler Relat Disord 2017; 20: 30-36.

26. Kermode AG, Tofts PS, Thompson AJ, et al. Heterogeneity of blood-brain barrier changes in multiple sclerosis: An MRI study with gadoliniumDTPA enhancement. Neurology 1990; 40: 229-235. 